\title{
1st Young Environmental Scientists Meeting (YES-Meeting)
}

\author{
New Challenges in Environmental Sciences <http://yes.sac-online.eu>
}

\author{
Markus Brinkmann • André Dabrunz $\cdot$ Thomas-Benjamin Seiler $\cdot$ Cornelia Kienle $\cdot$ Mirco Bundschuh
}

Erhalten: 22. November 2008/Akzeptiert: 12. Dezember 2008/Online veröffentlicht: 14. Januar 2009

(C) Springer-Verlag 2009

Der SETAC Europe Student Advisory Council (SAC) lädt vom 16. bis 18. März 2009 zum „1st Young Environmental Scientists Meeting“ (YES-Meeting) mit dem Titel „New Challenges in Environmental Sciences" auf den Campus Landau (Universität Koblenz-Landau) ein.

Seit seiner Gründung in Den Haag im Jahre 2006 vertritt der SAC die Belange der studentischen SETAC EuropeMitglieder. Schon während der Gründungssitzung am 9. Mai 2006 wurden viele Aspekte diskutiert, die in der Arbeit des SAC seither besondere Beachtung finden. So zählen wir vor allem die Verbesserung der Netzwerkstrukturen zwischen etablierten WissenschaftlerInnen und Nachwuchskräften, Informationsangebote zur Berufsplanung und Hilfestellungen bei der Teilnahme an den Jahrestagungen der SETAC Europe zu unseren wichtigsten Aufgaben.

Beim Young Scientist-Symposium „Meeting the new generation“, welches während der 17. Jahrestagung der SETAC Europe in Porto veranstaltet wurde, konnten Studierende in

Verantwortlicher Herausgeber: Henner Hollert

M. Brinkmann · T.-B. Seiler $(\bowtie)$

Institut für Umweltforschung, RTWH Aachen,

Worringerweg 1, 52074 Aachen, Deutschland

E-Mail: seiler@bio5.rwth-aachen.de

A. Dabrunz $\cdot$ M. Bundschuh

Institut für Umweltwissenschaften, Universität Koblenz-Landau,

Campus Landau, Fortstraße 7, 76829 Landau/Pfalz, Deutschland

E-Mail: bundschuh@uni-landau.de

C. Kienle

Ökotoxzentrum, EAWAG,

Überlandstrasse 133, 8600 Dübendorf, Schweiz

und

Abteilung Physiologische Ökologie der Tiere,

Universität Tübingen,

Konrad-Adenauer-Str. 20, 72070 Tübingen, Deutschland informeller Atmosphäre Kontakt zu jungen Kolleginnen und Kollegen aufnehmen. Zudem verfolgten die etwa 90 Teilnehmer fünf ausgewählte wissenschaftliche Präsentationen, erörterten Probleme und diskutierten Lösungen. Auch das Lunch-time-Seminar „A day in the life of ... - Career Talks" während der darauf folgenden Jahrestagung in Warschau war sehr gut besucht. Über 150 Studierende nahmen die einmalige Chance wahr, Einblicke in Lebensläufe, Fragen und Entscheidungen, sowie die notwendigen Fähigkeiten während der Berufsplanung der Gastredner zu erlangen. Der Erfolg beider Veranstaltungen ist unsere Motivation, weitere Schritte in diese Richtung zu unternehmen.

Das hier angekündigte YES-Meeting wird organisiert und durchgeführt von Studierenden für Studierende. Besonderer Wert wurde bei der Konzeption auf die speziellen Bedürfnisse junger WissenschaftlerInnen gelegt. So werden die Präsentationen aus zwei gleichbedeutenden Teilen (je 15 Minuten) bestehen: dem Vortrag und einer Diskussion, während der umfangreich auf die Belange des Redners eingegangen werden kann. Weiterhin wird Michael C. Newman (Virginia Institute for Marine Science) den TeilnehmerInnen während des Workshops „How to prepare excellent scientific publications" viele Tipps und Hinweise zum erfolgreichen Publizieren geben. Außerdem ist eine Veranstaltung geplant - ähnlich den „Career Talks“ von Warschau - die NachwuchswissenschaftlerInnen Möglichkeiten für die Karriereplanung aufzeigen soll. Hierzu werden den Studierenden Vertreter aus dem akademischen (Paul van den Brink, Alterra, SETAC Europe Präsident) und dem regulatorischen Bereich (Christine Fuell, EFSA) sowie der Industrie Einblick in die Qualitäten und Erfordernisse geben, die sie für ihren jeweiligen Werdegang als wichtig erachten. Während eines Job-Corners wird eingeladenen Unternehmen die Möglichkeit gegeben, sich den anwesenden Nachwuchskräften als potenzieller Arbeitgeber vorzustellen. 
Ein besonderer Dank gilt an dieser Stelle den großzügigen Sponsoren der Tagung, durch deren Hilfe es uns möglich sein wird, keine Tagungsgebühr erheben zu müssen und allen Vortragenden einen Reisekosten-Beitrag gewähren zu können. Weiterhin werden kostengünstige Unterbringungen zur Verfügung stehen.
Für weitere Informationen zum Student Advisory Council (SAC) besuchen Sie bitte unseren neuen Internetauftritt (http://www.sac-online.eu).

Gerne stehen wir auch für persönlichen Kontakt, Fragen und Anregungen zur Verfügung: setac_sac@yahoo.com. 